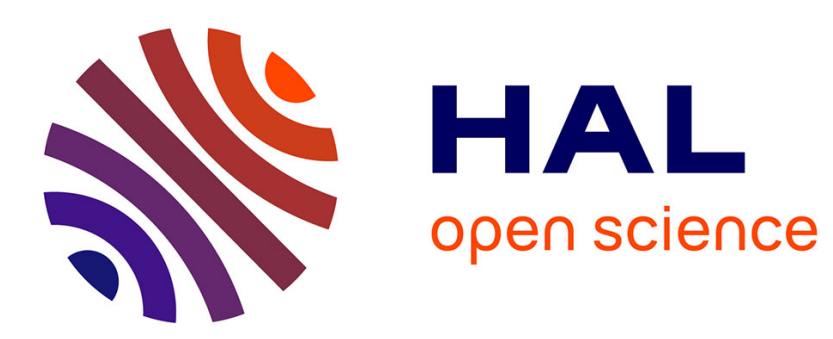

\title{
Plastic and viscous dissipations in foams: cross-over from low to high shear rates
}

Philippe Marmottant, Francois Graner

\section{To cite this version:}

Philippe Marmottant, Francois Graner. Plastic and viscous dissipations in foams: cross-over from low to high shear rates. Soft Matter, 2013, 9, pp.9602-9607. hal-00911660

\section{HAL Id: hal-00911660 \\ https://hal.science/hal-00911660}

Submitted on 29 Nov 2013

HAL is a multi-disciplinary open access archive for the deposit and dissemination of scientific research documents, whether they are published or not. The documents may come from teaching and research institutions in France or abroad, or from public or private research centers.
L'archive ouverte pluridisciplinaire HAL, est destinée au dépôt et à la diffusion de documents scientifiques de niveau recherche, publiés ou non, émanant des établissements d'enseignement et de recherche français ou étrangers, des laboratoires publics ou privés. 


\title{
Soft Matter
}

\section{PAPER}

Cite this: Soft Matter, 2013, 9, 9602

\section{Plastic and viscous dissipations in foams: cross-over from low to high shear rates}

\begin{abstract}
P. Marmottant ${ }^{* a}$ and F. Graner ${ }^{b}$
Soft glassy materials made of deformable cells, such as liquid foams, simultaneously display elastic, plastic and viscous behaviours. Bubble deformation is elastic until the material plastically yields and bubbles swap neighbours, then bubbles relax dissipatively towards a new energy minimum. This relaxation occurs in a finite time, and shearing a foam at a fast strain rate compared to that time leads to a viscous flow. To describe such an elastic, plastic and viscous behaviour we introduce a simplified scalar model of foam deformation and flow with a periodic pinning potential. The continuum mechanics behaviour of the foam emerges as an ensemble average over disordered units without requiring that they are coupled. Our model captures surprisingly well various features of the viscous dissipation during plastic deformation. At low shear rates, the time averaged stress is smaller than the static yield stress. A critical shear rate exists: any flow at fixed stress has a shear rate above this critical value. Moreover, the model only involves measurable parameters, which enables us to compare it with existing experiments and simulations.
\end{abstract}

Received 2nd May 2013

Accepted 9th August 2013

DOI: $10.1039 / \mathrm{c} 3 \mathrm{sm} 51220 \mathrm{~g}$

www.rsc.org/softmatter
Rouyer et $a l .{ }^{8}$ have published the non-linear response (high harmonics) of foams subjected to an oscillatory shear. They have demonstrated that these experimental data are a strong benchmark against which to test theoretical models. They have compared two classes of models which predict the foam behaviour in steady and transient flows at various time scales and amplitudes. Each approach has partial successes and brings its own contribution to understanding. First is the soft glassy rheology model ${ }^{9}$ in which groups of bubbles are characterised by

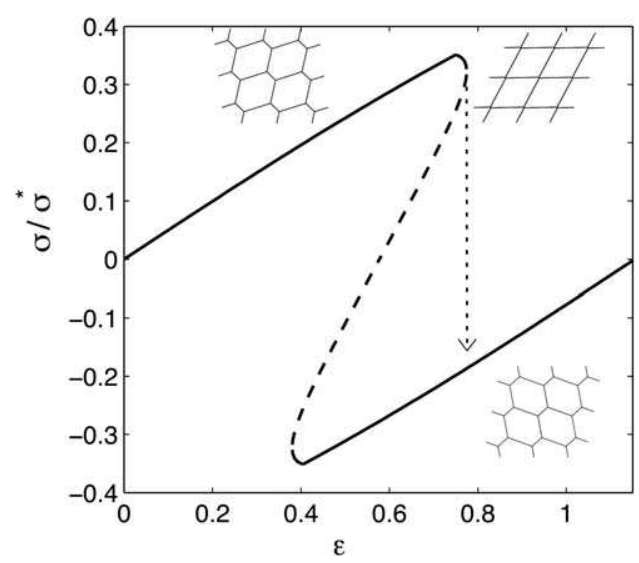

Fig. 1 Elastic stress $\sigma$ versus strain $\varepsilon$ for an ordered foam. Quasistatic response obtained by Princen's exact calculation for $1 \%$ liquid fraction. ${ }^{6}$ The stress $\sigma$ is expressed in units of the characteristic stress $\sigma^{*}$, which is the bubble surface tension divided by the bubble radius. The dashed part of the curve is unstable, so that at increasing $\varepsilon$ the actual response is indicated by the thin dotted arrow. The geometry of the bubble walls at three points of the curve is shown as insets. 
a local strain and energy density, and are coupled to their neighbors and yield stochastically. With few parameters it fits well the linear and non-linear oscillatory foam response. ${ }^{8}$ The second approach is phenomenological models, in which a foam is described as a visco-elasto-plastic with experimentally measurable parameters. ${ }^{10-13}$ They successfully predicted space variations of foam velocity, deformation and rearrangement fields in various flow geometries, in the quasistatic regime ${ }^{14}$ and at finite velocity (see ref. 15 for review).

To contribute to the global picture, the goal of the present paper is threefold. First, we want to link actually measurable properties at the bubble level with the flow predictions at the global scale of the whole foam. Second, we want to determine whether simply averaging over individual discrete units, without coupling them, could be enough to see the continuous behaviour emerge at a large scale. Third, we want to understand theoretically the cross-over between behaviours at quasistatic and finite shear rates, which has already been characterized in experiments. ${ }^{16}$

For that purpose, we propose a model to merge the micromechanical approach ${ }^{6,7}$ and the phenomenological one (both in quasistatic ${ }^{12}$ and finite shear rate regimes ${ }^{11}$ ) into a single coherent picture. We use a minimal set (which can be refined later) of experimentally measurable parameters. We first consider an ordered foam, and then a disordered one. We focus on the nonlinear response of the foam at various amplitudes. We compare the model predictions with existing simulations or experiments.

\section{The phenomenological model for ordered foam plasticity}

To reproduce the quasistatic curve of Fig. 1 and include viscosity as well, we suggest using a local scalar model. It involves three simple ingredients (Fig. 2).

The first one is an internal variable $\varepsilon_{\mathrm{e}}$ for the elastic deformation, representing the distortion of bubbles away from the relaxed state. The physical variables are thus the strain $\varepsilon$, defined as the time integral of $\dot{\varepsilon}$; the elastic deformation $\varepsilon_{\mathrm{e}}$, which is a function of state; and the irreversible plastic deformation, $\varepsilon_{\mathrm{p}}$, defined as their difference: $\varepsilon=\varepsilon_{\mathrm{e}}+\varepsilon_{\mathrm{p}}$.

How the applied (i.e. total) strain $\varepsilon$ is split between $\varepsilon_{\mathrm{e}}$ and $\varepsilon_{\mathrm{p}}$ raises the question of what precisely determines $\varepsilon_{\mathrm{p}}$ : predicting it will be the object of the present model. The elastic modulus associated with $\varepsilon_{\mathrm{e}}$ is denoted as $E$.

The second is a dissipation $\eta$ affecting the plasticity relaxation $\dot{\varepsilon}_{\mathrm{p}}$, that is, the motion of bubbles sliding past each other. It will be interpreted below as the extensional viscosity of bubble walls. This viscosity $\eta$ can be non-linear, ${ }^{17}$ for instance if it depends on the shear rate. The corresponding internal relaxation time is $\tau=$ $\eta / E$, which might be physically more relevant than $\eta$ and is often easier to measure. For instance, in the case of dry foams, Biance et al. ${ }^{18}$ have shown using 3D bubble clusters as a model system that $\tau$ scales as the ratio of dilational surface viscosity and surface tension. Durand et al. ${ }^{19}$ have evidenced and explained the impact of interfacial elasticity and viscosity on $\tau$ in dry 2D foams. In the case of wet foams with mobile interfaces, Le Merrer $e t a l .^{20}$ have studied rearrangements induced by coarsening; they showed that $\tau$ scales in this case as the ratio of bulk foaming liquid (a)

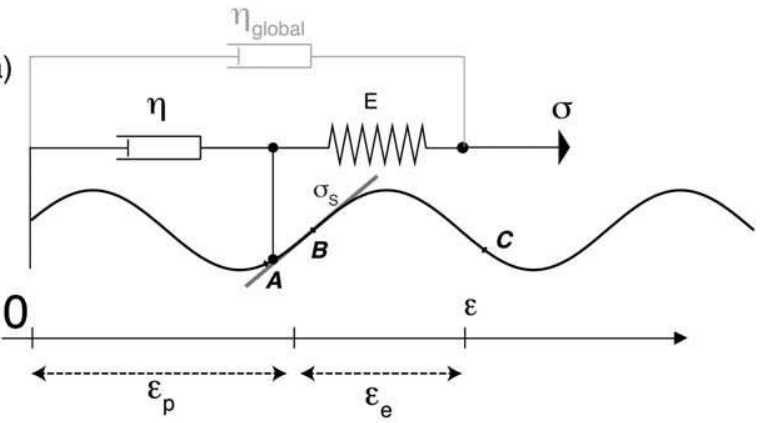

(b)

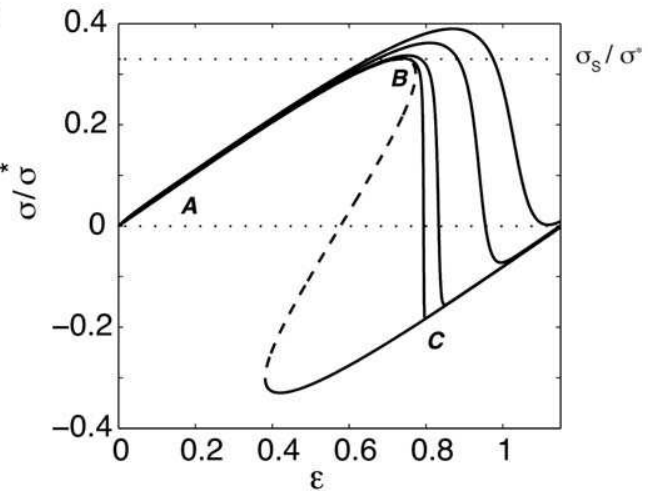

Fig. 2 Phenomenology of an ordered foam. (a) Mechanical model of eqn (2): the dashpot stands for the viscous dissipation $\eta$, the spring for the elastic modulus $E$, and the sinusoidal curve for the periodic potential (last term of eqn (1)). It is possible to add a global scale viscosity, $\eta_{\text {global }}{ }^{12}$ (in grey). Other notations are explained in the text. (b) Elastic stress $\sigma$ versus strain $\varepsilon$ for the model at increasing shear rates (eqn (2) or (3)). From left to right: $\dot{\varepsilon} \tau=0,0.002,0.01,0.05$ and 0.1 . The model parameters are chosen to match closely the curve in Fig. 1 when $\dot{\varepsilon} \tau=0: E=$ $0.74 \sigma^{*}, \sigma_{\mathrm{s}}=0.33 \sigma^{*}, \varepsilon_{0}=2 / \sqrt{3}$. Letters A, B, C establish the correspondence between both panels.

viscosity and osmotic pressure, with a dimensionless coefficient depending on the liquid fraction.

The third is a potential which pins the plastic deformation $\varepsilon_{\mathrm{p}}$ around discrete positions (Fig. 2a). This has already been introduced to account for stick-slip motions, ${ }^{\mathbf{2 1 , 2 2}}$ then later in plasticity studies (see for instance ref. 23, the formalism of which we adapt here). Phenomenologically we write this effective potential with a period $\varepsilon_{0}$ :

$$
U_{\text {eff }}\left(\varepsilon, \varepsilon_{\mathrm{p}}\right)=\frac{E}{2}\left(\varepsilon-\varepsilon_{\mathrm{p}}\right)^{2}+\sigma_{\mathrm{s}} \frac{\varepsilon_{0}}{2 \pi} \cos \left(\frac{2 \pi \varepsilon_{\mathrm{p}}}{\varepsilon_{0}}\right) .
$$

The discrete positions are local potential minima. In the quasistatic limit, the plastic deformation always relaxes to one of these positions. There is a yield strain $\varepsilon_{\mathrm{s}}$, where the subscript s stands for "static", as it will be opposed below to a "dynamic" yield strain. Equivalently we can introduce the associated yield stress $\sigma_{\mathrm{s}}=E \varepsilon_{\mathrm{s}}$. It conveys the same information as $\varepsilon_{\mathrm{s}}$ if the elasticity is linear, which we temporarily assume for simplicity. It is visible in Fig. 2a as the maximum slope of the potential.

In the dynamic regime, plastic deformation can differ from these discrete positions and take continuous values. Its evolution equation is obtained by injecting $U_{\text {eff }}$ into the dissipation function formalism ${ }^{24,25}$ as in eqn (6) and (7) of ref. 11: 


$$
\eta \dot{\varepsilon}_{\mathrm{p}}=-\frac{\partial U_{\text {eff }}\left(\varepsilon, \varepsilon_{\mathrm{p}}\right)}{\partial \varepsilon_{\mathrm{p}}}=E\left(\varepsilon-\varepsilon_{\mathrm{p}}\right)-\sigma_{\mathrm{s}} \sin \left(\frac{2 \pi \varepsilon_{\mathrm{p}}}{\varepsilon_{0}}\right) .
$$

In eqn (2) the plasticity rate $\dot{\varepsilon}_{\mathrm{p}}$ can be finite even if $\sigma<\sigma_{\mathrm{s}}$. The plasticity rate can even be temporarily negative if the plastic deformation relaxes back to a pinning minimum, for instance when relieving a small, positive stress as in Fig. 2 a.

In the quasistatic regime, $\varepsilon_{\mathrm{p}}$ increases with time by discrete steps: it is a staircase (piecewise constant) and its time derivative is a Dirac comb. The shape of the quasistatic response curve is thus obtained by setting $\dot{\varepsilon}_{\mathrm{p}}=0$ in eqn (2). The solution is the dashed curve in Fig. 2b. The sine in eqn (2) creates a slight curvature in the initial elastic regime, thus limiting the linear elasticity range. All other features of the curve are very similar to the Princen model for a dry foam (Fig. 1).

If we apply a constant, finite shear rate $\dot{\varepsilon}$, the solutions of eqn (2) are displayed in Fig. $2 b$ for increasing values of $\dot{\varepsilon}$. We can use the value of $\dot{\varepsilon}$ to define a non-dimensional time $t^{\prime}=\dot{\varepsilon} t$ and accordingly $\dot{\varepsilon}_{\mathrm{p}}^{\prime}=\dot{\varepsilon}_{\mathrm{p}} / \dot{\varepsilon}$. Eqn (2) then becomes:

$$
\dot{\varepsilon} \tau \dot{\varepsilon}_{\mathrm{p}}^{\prime}=\varepsilon-\varepsilon_{\mathrm{p}}-\varepsilon_{\mathrm{s}} \sin \left(\frac{2 \pi \varepsilon_{\mathrm{p}}}{\varepsilon_{0}}\right) .
$$

The model is therefore governed by three nondimensional numbers representing: the stretching rate $\dot{\varepsilon} \tau$, the static yield strain $\varepsilon_{\mathrm{s}}$, and the period $\varepsilon_{0}$ of the pinning potential.

Our model (eqn (2) or (3)) has similarities with the Coussot and Ovarlez model ${ }^{26}$ based on a stress function which increases with time and then instantaneously jumps to a smaller value. However, in their model, a phenomenological waiting time with zero stress is introduced after the jump, to account for internal relaxation. Here, we do not impose the waiting time, which results from the stress relaxation via the viscosity $\eta$. Eqn (3) was used in various contexts, but not yet for foams: it is known as the Prandtl-Tomlinson model for stick-slip, ${ }^{27}$ and describes Josephson junctions ${ }^{28}$ or the driven pendulum.

\section{From a quasistatic to a dynamic regime}

\subsection{Time averaged stress versus yield stress}

At a small shear rate, $\dot{\varepsilon} \tau \ll 1$, the stress exhibits sudden drops from the static yield stress value $\sigma_{\mathrm{s}}$ to a value $\sigma_{\mathrm{s}}-\Delta \sigma$. By definition of the quasistatic regime, the stress is completely relaxed before the next drop occurs (see Fig. 3), and $\Delta \sigma \simeq E \varepsilon_{0}$. In practice, since the linear elasticity regime is limited by the convexity of the $\sigma(\varepsilon)$ curve (Fig. 2b), $\Delta \sigma$ is slightly smaller than $E \varepsilon_{0}$. We note $\bar{\sigma}$, the time average of $\sigma$. In the quasistatic steady shear regime $(\dot{\varepsilon} \tau \rightarrow 0) \bar{\sigma} \simeq \sigma_{\mathrm{s}}-\Delta \sigma / 2$. This defines the dynamical yield stress

$$
\sigma_{\mathrm{d}} \simeq \sigma_{\mathrm{s}}-\Delta \sigma / 2
$$

which is thus lower than the static one: $\sigma_{\mathrm{d}}<\sigma_{\mathrm{s}}$. Here there is an analogy with solid friction, where the quasistatic solid friction coefficient is smaller than the static one, inducing stick-slip oscillations ${ }^{21}$ Note that the static yield stress $\sigma_{\mathrm{s}}$ is an ingredient of the model, while the dynamic one $\sigma_{\mathrm{d}}$ is an output.

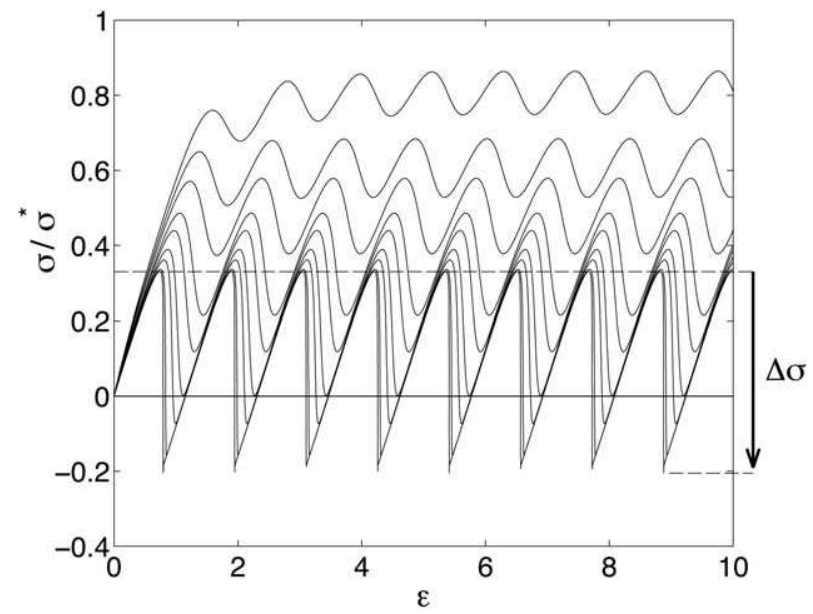

Fig. 3 Elastic stress versus strain for solutions of eqn (2) or (3). Dimensionless numbers are $\varepsilon_{0}=2 / \sqrt{3}, \varepsilon_{\mathrm{s}}=0.4459$ (corresponding to $\sigma_{\mathrm{s}}=0.33 \sigma^{*}$ ), and shear rates increasing from bottom to top: $\dot{\varepsilon} \tau=0.002,0.01,0.05,0.1,0.2,0.3,0.5,0.7$ and 1. The horizontal continuous line indicates a stress value 0 , while dashes indicate values $\sigma_{\mathrm{s}}$ and $\sigma_{\mathrm{s}}-\Delta \sigma$, where $\Delta \sigma$ is the quasistatic (i.e. the highest possible) amount of stress relaxation during a plastic event.

Conversely, at larger $\dot{\varepsilon} \tau$, the relaxation is not complete when a new drop occurs. The time average value of elastic stress (plotted in Fig. 4) now includes a contribution increasing with $E \dot{\varepsilon} \tau$. We can write it as $\eta \dot{\varepsilon}$ with $\eta=E \tau$; see below the interpretation of this term. It implies that Fig. 4 admits the same asymptote as both the Puglisi and Truskinovsky analytical calculation ${ }^{23}$ and the classical Bingham viscoplastic equation, ${ }^{29}$ $\bar{\sigma}(\dot{\varepsilon}) \simeq \sigma_{\mathrm{d}}+\eta \dot{\varepsilon}$.

Fig. 4 indicates that $\bar{\sigma}$ increases with $\dot{\varepsilon} \tau$ beyond $\sigma_{\mathrm{d}}$. This is also the case with the spatio-temporal model of Picard et al. ${ }^{30}$ Kraynik and Hansen ${ }^{7}$ too obtained a qualitatively similar result (the only difference is the discontinuities on the stress versus strain curve which appears in their solutions and not in ours). They

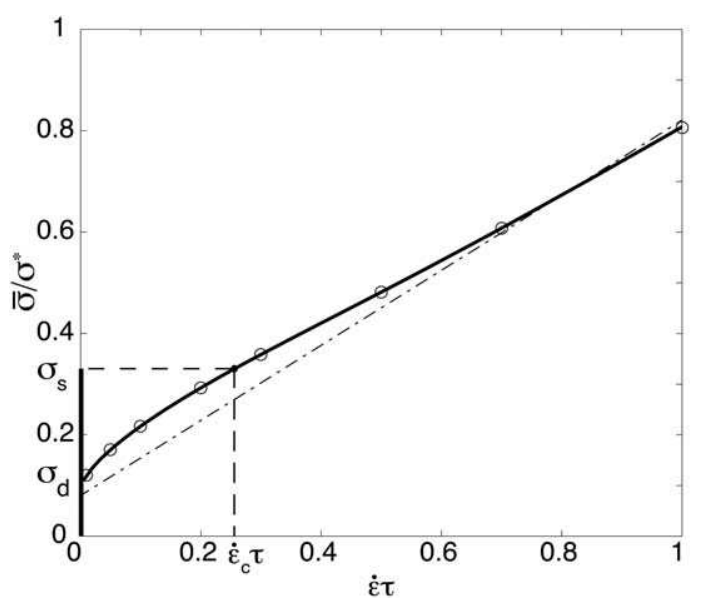

Fig. 4 Cross-over from a quasistatic to a dynamic regime. Solid line: time averaged stress $\bar{\sigma}$ obtained from curves presented in Fig. 3, versus adimensioned shear rate $\dot{\varepsilon} \tau$. Circles: plateau values of stress, calculated after phase-averaging over $N=$ 100 elements for a deformation of $\varepsilon=6$ (right axis of Fig. 5b). Dashed dotted line: the Bingham model, plotted for comparison. 
performed a detailed geometrical computation in an ordered foam, where stretching films of thickness $h$ are affected by an effective extensional viscosity $\mu_{\text {bulk }} h / R$. In their model, $\mu_{\text {bulk }}$ is the bulk solution viscosity and $R$ the bubble radius. Here we capture the essence of the foam behaviour using minimal ingredients, without space dependence, and without the requirement to include in the elasticity any detail of bubble geometry.

Overall the model predicts that, in a steady shear flow, the time average $\bar{\sigma}$ of the instantaneous elastic stress $\sigma=E \varepsilon_{\mathrm{e}}$ can be either smaller or larger than $\sigma_{\mathrm{s}}$ : see below the discussion regarding disordered foams.

\subsection{Fixed stress versus fixed strain rate}

Experimentally, fixing stress or strain rate leads to different behaviours. The simplest case is fixed $\dot{\varepsilon}$ : any value of $\dot{\varepsilon}>0$ determines one and only one $\bar{\sigma}$ (Fig. 4), $\sigma$ and $\varepsilon_{\mathrm{e}}$ oscillate periodically in time, $\varepsilon_{\mathrm{p}}$ and $\varepsilon$ become arbitrarily large and the foam flows.

At fixed $\sigma$, two cases occur (Fig. 3). For $\sigma<\sigma_{\mathrm{s}}, \varepsilon_{\mathrm{e}}$ and $\varepsilon$ remain finite and below $\varepsilon_{\mathrm{s}}$. Conversely, for $\sigma>\sigma_{\mathrm{s}}, \varepsilon_{\mathrm{p}}$ and $\varepsilon$ becoming arbitrarily large, the foam flows, and $\bar{\sigma}$ is the time average plotted in Fig. 4.

The richest situation occurs at fixed $\bar{\sigma}$, with three distinct possibilities (Fig. 4). For any given $\bar{\sigma}$ value below $\sigma_{\mathrm{d}}$, there exists only one shear rate, the truly static value $\dot{\varepsilon}=0$. For any given $\bar{\sigma}$ value between $\sigma_{\mathrm{d}}$ and $\sigma_{\mathrm{s}}$, two shear rates can coexist: one is static, $\dot{\varepsilon}=0$, and the other is a slow shear rate, $\dot{\varepsilon}<\dot{\varepsilon}_{\mathrm{c}}$, that is stable because the slope of the stress versus strain curve is positive. ${ }^{31}$ For any given $\sigma$ value above $\sigma_{\mathrm{s}}$, there exists only one shear rate, in the dynamic regime, $\dot{\varepsilon}>\dot{\varepsilon}_{\mathrm{c}}$. Here $\dot{\varepsilon}_{\mathrm{c}}$ is the critical shear rate marking the cross-over between the quasistatic regime, $0<\dot{\varepsilon} \ll \dot{\varepsilon}_{\mathrm{c}}$, and the dynamic regime, $\dot{\varepsilon} \gg \dot{\varepsilon}_{\mathrm{c}}$. For ordered foams, this value is finite and measurable. Using Fig. 4, we determine the value of $\dot{\varepsilon}_{\mathrm{c}}$ :

$$
\dot{\varepsilon}_{\mathrm{c}} \tau=0.26 \text {. }
$$

To summarize, the main results of the present paper are: (i) the existence of $\dot{\varepsilon}_{\mathrm{c}}$ above which the foam flows above the static yield stress; (ii) the possibility to predict with such a local and simple model the vertical line segment in Fig. 4 , with $\sigma_{\mathrm{s}}>\sigma_{\mathrm{d}}$, opening the possibility of spatial coexistence between static and flowing shear bands $;^{26}$ and (iii) the prediction of $\bar{\sigma}(\dot{\varepsilon})$ (solid line of Fig. 4) which enables to calculate $\dot{\varepsilon}_{\mathrm{c}}$ (eqn (5)). Note that the Bingham model is on the right of the solid line of Fig. 4. It thus yields a slightly overestimated prediction of $\dot{\varepsilon}_{\mathrm{c}}, \dot{\varepsilon}_{\mathrm{c}} \sim \frac{\Delta \sigma}{2 \eta} \sim \frac{\varepsilon_{0}}{2 \tau}$, here $\dot{\varepsilon}_{\mathrm{c}} \sim 0.35 / \tau$. Its order of magnitude is correct enough to be used in practice.

\section{Disordered foams}

\subsection{Cross-over between quasistatic and dynamic models}

For disordered foams, we implement in parallel $N$ elements, each being described by eqn (3), a random distribution in any of the model parameters. We now discuss the ensemble average over phase disorder (averaging over $\varepsilon_{0}$ or $\varepsilon_{\mathrm{s}}$ yields relevant predictions too, data not shown). The average stress $\langle\sigma\rangle$ is over an ensemble of $N$ foams with different phases $\phi$. In practice, in eqn (2), the term $\sin \left(2 \pi \varepsilon_{\mathrm{p}} / \varepsilon_{0}\right)$ is replaced with $\sin \left(2 \pi \varepsilon_{\mathrm{p}} / \varepsilon_{0}+\phi\right)$ where phases $\phi$ are evenly distributed in the interval $[0 ; 2 \pi]$. Spatial models which include non-affine movements (probably important to understand the foam local rheological properties $^{32}$ ) statistically describe the local disorder and result in a similar averaging.

We can now bridge the gap between two predictive descriptions of foam flows. One, with a rate-independent response and elements never stretched over yield $\left(\varepsilon_{\mathrm{e}} \leq \varepsilon_{\mathrm{s}}\right)$, is valid in the "quasistatic" limit $\dot{\varepsilon} \tau \rightarrow 0 .{ }^{12}$ The other, with a viscous behaviour and elements that can overstretch $\left(\varepsilon_{\mathrm{e}}>\varepsilon_{\mathrm{d}}\right)$, is valid in the dynamic regime ( $\dot{\varepsilon}$ finite). ${ }^{11}$

In quasistatic shear (Fig. 5a), when $N$ increases, the elastic modulus is mostly unchanged (apart from a small diminution due to the convexity of the $\sigma(\varepsilon)$ curve); period and amplitude of oscillations decrease as $1 / N$. At large $N$ the stress undergoes tiny fluctuations around its average, which justifies the description of a foam as a continuous medium. ${ }^{\mathbf{1 4}}$ The onset of plasticity becomes abrupt: the slope breaks at $\varepsilon_{\mathrm{d}}$ between the linear elastic regime $\sigma=E \varepsilon$ and the plastic plateau $\sigma=\sigma_{\mathrm{d}}$. By averaging over different populations (having an abrupt plasticity with a different yield strain) we model a progressive onset of plasticity and thus recover our preceding model. ${ }^{12}$
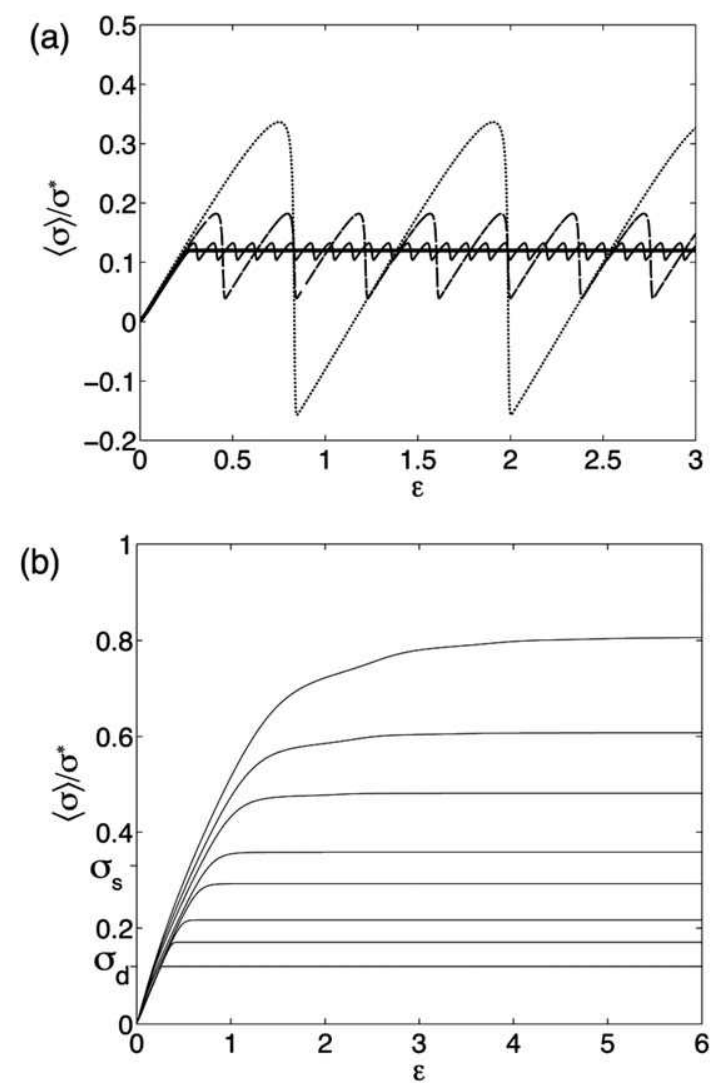

Fig. 5 Disorder in phase. (a) Ensemble average $\langle\sigma\rangle$ is performed over an increasing number $N$ of elements with random phases. From thinner to thicker curves: $N=1$ (dots), 3 (dashes), 11 (thin line), 100 (thick line). Motion is quasistatic, $\dot{\varepsilon} \tau=0.01$. (b) Same ensemble average, performed over $N=100$ elements, with a shear rate increasing from bottom to top: same values of $\dot{\varepsilon} \tau$ as in Fig. 3 . Curves for $\dot{\varepsilon} \tau=0.002$ and 0.01 are indistinguishable. 
At finite shear rates, for spatially extended foams, a mean field approach $^{30,33,34}$ extends the above description. In fact, let $N_{\text {corr }}$ be the number of correlated bubbles involved in each stress relaxation (the case of ordered foams would correspond to $N_{\text {corr }}=N$ ). The stress drop of the average is $\sim \Delta \sigma N_{\text {corr }} / N$ and eqn (5) becomes

$$
\begin{aligned}
& \dot{\varepsilon}_{\mathrm{c}} \tau \simeq \frac{\varepsilon_{0}}{2} \frac{N_{\text {corr }}}{N}, \\
& \sigma_{\mathrm{s}}-\sigma_{\mathrm{d}} \simeq \frac{\Delta \sigma}{2} \frac{N_{\text {corr }}}{N} .
\end{aligned}
$$

For disordered foams, $N_{\text {corr }} \ll N$, stress drops become much smaller than $\sigma_{\mathrm{s}}$, so that $\sigma_{\mathrm{d}} \simeq \sigma_{\mathrm{s}}$. Fig. $5 \mathrm{~b}$ then becomes equivalent to Saramito's continuous models ${ }^{11,17}$ which we thus link with a simplified micromechanical description. The ensemble average over phase disorder (Fig. 5b) yields the same results as the time average (see circles in Fig. 4).

In both cases (ensemble average or time average), the averaged stress is always larger than $\sigma_{\mathrm{d}}$ in steady flow. However, these averages can be either lower or higher than $\sigma_{\mathrm{s}}$. Indeed they are performed over time intervals (in the case of time average) or over elements (in the case of ensemble average) which are partly below $\sigma_{\mathrm{s}}$ and partly above it, in variable proportion. This proportion, and thus the value of $\sigma$, depends on $\dot{\varepsilon} \tau$ rather than on $N_{\text {corr }} / N$. It makes possible for a steady flow to be below $\sigma_{\text {s }}$, and thus for flowing and static states to coexist at the same stress.

Note that Fig. 4 describes only the elastic contribution to the stress $\sigma$ and does not describe any viscous term. Here $\eta=E \tau$ originates from an elastic modulus and the internal time characteristic of plasticity rate, $\dot{\varepsilon}_{\mathrm{p}}=\left(\varepsilon_{\mathrm{e}}-\varepsilon_{\mathrm{d}}\right) / \tau$. Thus $\eta$ plays a role because it slows down the bubble shape relaxation, thus increasing the average bubble deformation, and thus increasing the average elastic deformation. The total stress could also include an additional truly dissipative term (viscous friction in the interstitial fluid linked to the affine part of deformation), proportional to the global viscosity $\eta_{\text {global }}$ (Fig. 2), which is relevant at any $\sigma$ as long as $\dot{\varepsilon} \neq 0$, but is small (while $\eta$, although stronger, is relevant only above $\left.\sigma_{\mathrm{d}}\right){ }^{\mathbf{1 1}}$

As expected, we obtain the classical viscoplastic Bingham model in the steady shear regime. In fact, in this limit, the elastic deformation $\varepsilon_{\mathrm{e}}$ is constant; it can thus be eliminated and the stress expressed versus $\dot{\varepsilon}$ only. Except for this limit, in the general case the full visco-elasto-plastic description is necessary. ${ }^{15}$

\subsection{Tests of the model}

To test the prediction of eqn (5), we can perform a comparison with numerical simulations by Picard et $a .^{30}$ of elastoplastic elements with a relaxation time, $\tau=\eta / E$ in our notations. They measure $\sigma_{\mathrm{s}}-\sigma_{\mathrm{d}}$ of the order of $0.25 \sigma_{\mathrm{s}}$. In our notations, this yields the stress drop (from $\Delta \sigma / 2 \simeq \sigma_{\mathrm{s}}-\sigma_{\mathrm{d}}$ ), and we predict $\dot{\varepsilon}_{\mathrm{c}} \sim$ $\Delta \sigma / 2 \eta \simeq 0.25 \sigma_{\mathrm{s}} / E \tau$. Such prediction is comparable to the observed value ${ }^{30} \dot{\varepsilon}_{\mathrm{c}}=0.3 \sigma_{\mathrm{s}} / E \tau$.

We now discuss a possible experimental determination of the link between stress drops and localisation. Dennin and coworkers shear a bubble raft in a 2D cylindrical Couette geometry (with a gap of $\sim 15$ to 22 bubble diameters): they observe shear localisation, ${ }^{35}$ which is favoured by the heterogeneous stress (for review and discussion, see ref. 5 and 15). The static yield strain $^{36}$ is $\varepsilon_{\mathrm{s}} \simeq 0.12$. The relaxation time is widely distributed between $0.1 \mathrm{~s}^{-1}$ and $10 \mathrm{~s}^{-1}$, with a typical order of magnitude ${ }^{37} \tau \sim 1 \mathrm{~s}^{-1}$. The stress drops ${ }^{36,37}$ are typically $\Delta \sigma \simeq 0.2 \sigma_{\mathrm{s}}$. Our model predicts $\dot{\varepsilon}_{\mathrm{c}}=\Delta \sigma / \sigma_{\mathrm{s}} \times \varepsilon_{\mathrm{s}} / 2 \tau \simeq 0.01 \mathrm{~s}^{-1}$. This order of magnitude corresponds to their observed value, ${ }^{35}$ around $0.01-0.07 \mathrm{~s}^{-1}$.

Finally, Fig. 6 compares our predictions with non-linear rheology experiments of foams under sinusoidal strain, ${ }^{\mathbf{8}}$ $\varepsilon=\varepsilon_{0} \sin \omega t$. Lissajous plots (Fig. 6a) at increasing amplitude display the expected ${ }^{8}$ transition from ellipses, reflecting a
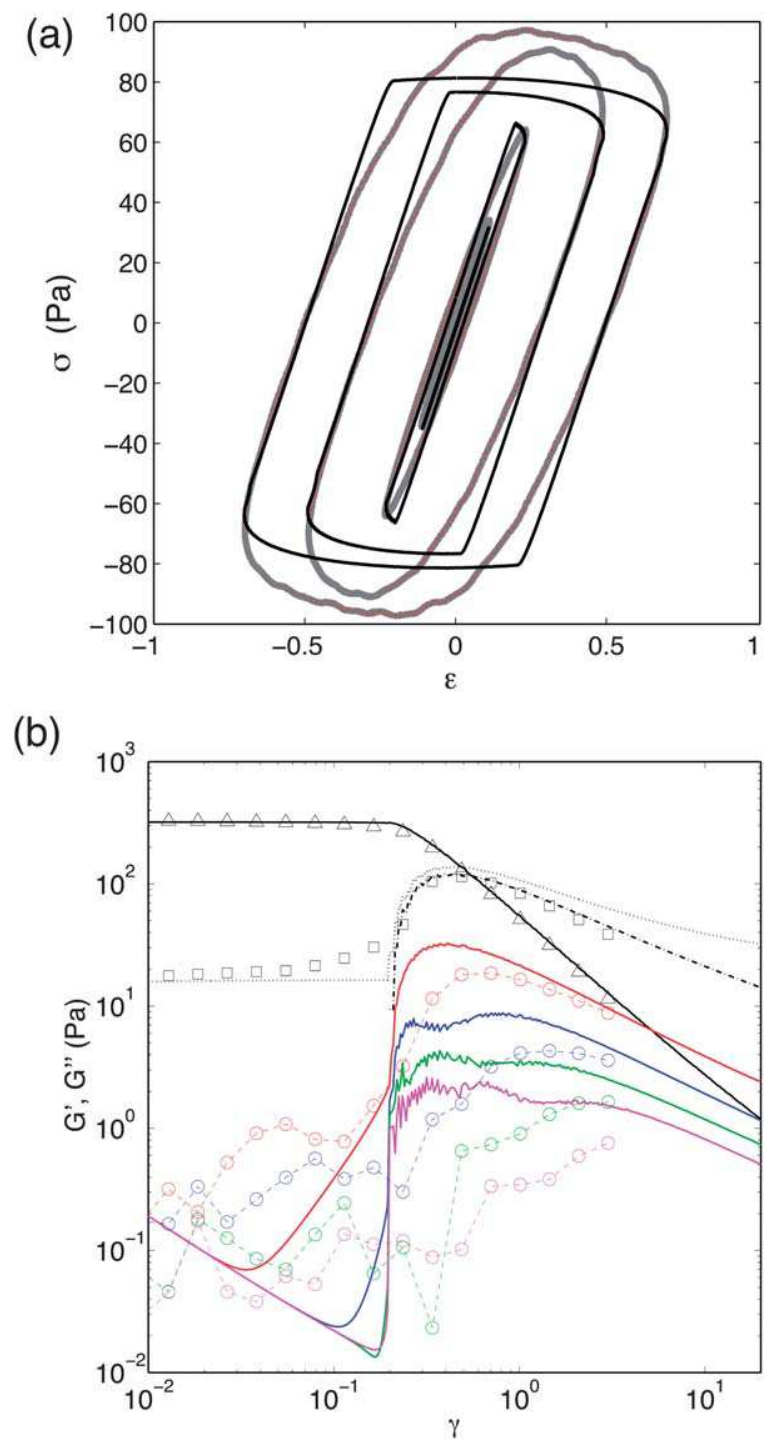

Fig. 6 Comparison with the nonlinear rheology of foam under oscillatory strain. ${ }^{8}$ (a) Lissajous plot of the stress versus the strain, parametrized by the time. Thick gray line: AOK foam experiments. ${ }^{8}$ The amplitudes of the applied oscillating strain were $0.11,0.23,0.49$ and 0.7 . Thin black line: present model with an ensemble average over $N=100$ phases; same parameters as before, and $\sigma^{*}=320 / 0.7=$ $457 \mathrm{~N} \mathrm{~m}^{-1}$ to match the experimental modulus $\mathrm{G}^{\prime}=320 \mathrm{~N} \mathrm{~m}^{-1}$, with a viscosity $\eta$ such that at $\varepsilon_{0}=1, \eta \dot{\varepsilon}_{\max }=\eta \omega=0.02$. (b) Log-log plot of the first harmonic (triangles: $G^{\prime}$ in phase, squares: $G^{\prime \prime}$ out of phase) and the four higher harmonics (red circles: 3rd harmonic, blue circles: 5th, green circles: 7th, pink circles: 9th). Symbols: experiments on AOK foam at $1 \mathrm{~Hz} .^{8}$ Lines: model, same parameters as in (a). Dots: with the addition of the global viscosity. 
viscous dissipation, to parallelograms, reflecting plastic dissipation. They are almost indiscernible from those obtained from our previous quasistatic model ${ }^{12}$ (not shown), the results of which are close to those of a simple elasto-plastic model such as that of ref. 8. We also plot the harmonics (Fig. 6b): the first harmonic ( $G^{\prime}$ in phase, $G^{\prime \prime}$ out of phase) and higher odd harmonics, the even ones being zero by symmetry. The stress is decomposed in a Fourier series that we write ${ }^{38}$ as $\sigma(t)=\varepsilon_{0} \sum_{n=1}^{\infty}\left[G_{n}^{\prime} \sin (n \omega t)+G_{n}^{\prime \prime} \cos (n \omega t)\right]$. The amplitude of the harmonic of order $n$ is defined as $\left|G_{n}^{*}\right|=\left[\left(G_{n}^{\prime}\right)^{2}+\left(G_{n}^{\prime \prime}\right)^{2}\right]^{1 / 2}$. As for our previous quasistatic model, ${ }^{12}$ the low-amplitude viscous dissipation is well described if and only if we include a global viscosity: this confirms that elasto-plasticity by itself is insufficient $^{8}$ while visco-elasto-plasticity is predictive. ${ }^{15,17}$

\subsection{Perspectives}

The model could be refined in two directions to improve the agreement with experiments. (i) Adding non-linear terms in the elasticity or viscosity, for instance shear-thinning. ${ }^{1}$ We would recover ref. 17, and asymptotically the Herschel-Bulkley model in steady shear. We do not anticipate major corrections to the present work, which already accounts for several sources of nonlinearity. (ii) Including $\dot{\varepsilon}$ which changes sign, or even orientation, yielding a tensorial model. Another perspective would be to model the linear response of the foam, for instance the wellknown frequency dependence of elastic and dissipative moduli. Finally, a last direction of research would be to investigate whether the existence of two different yield stresses, $\sigma_{\mathrm{s}}$ and $\sigma_{\mathrm{d}}$, determines possible avalanche-like behaviours.

\section{Acknowledgements}

We thank P. Saramito and J.-L. Barrat for discussions, C. Gay and F. Rouyer for critical reading of the manuscript, M. Dennin for references and comments on his experiments, F. Rouyer for providing raw data of ref. 8, and an anonymous referee for suggestions. Both teams belong to the CNRS consortiums "GdR Mousses et Emulsions", which we thank for financial support, and "GdR CellTiss".

\section{References}

1 D. Weaire and S. Hutzler, The physics of foams, Oxford University Press, Oxford, 1999.

2 I. Cantat, S. Cohen-Addad, F. Elias, F. Graner, R. Höhler, O. Pitois, F. Rouyer and A. Saint-Jalmes, Les mousses, Structure et dynamique, Belin, 2010, English translation: ed. S. J. Cox, Foams: Structure and Dynamics, translated by R. Flatman, Oxford University Press, 2013.

3 R. Höhler and S. Cohen-Addad, J. Phys.: Condens. Matter, 2005, 17, R1041-R1069.

4 N. D. Denkov, S. Tcholakova, K. Golemanov, K. P. Ananthpadmanabhan and A. Lips, Soft Matter, 2009, 5, 3389-3408.
5 G. Ovarlez, S. Cohen-Addad, K. Krishan, J. Goyon and P. Coussot, J. Non-Newtonian Fluid Mech., 2013, 193, 68-79. 6 H. M. Princen, J. Colloid Interface Sci., 1983, 91, 160-175.

7 A. M. Kraynik and M. G. Hansen, J. Rheol., 1987, 31, 175.

8 F. Rouyer, S. Cohen-Addad, R. Höhler, P. Sollich and S. Fielding, Eur. Phys. J. E, 2008, 27, 309-321.

9 P. Sollich, F. Lequeux, P. Hebraud and M. E. Cates, Phys. Rev. Lett., 1997, 78, 2020-2023.

10 V. Labiausse, PhD thesis, Université de Marne-la-Vallée, 2004.

11 P. Saramito, J. Non-Newtonian Fluid Mech., 2007, 145, 1.

12 P. Marmottant and F. Graner, Eur. Phys. J. E, 2007, 23, 337347.

13 S. Bénito, C. Bruneau, T. Colin, C. Gay and F. Molino, Eur. Phys. J. E, 2008, 25, 225-251.

14 P. Marmottant, C. Raufaste and F. Graner, Eur. Phys. J. E, 2008, 25, 371-384.

15 I. Cheddadi, P. Saramito and F. Graner, J. Rheol., 2012, 56, 213-239.

16 F. Rouyer, S. Cohen-Addad, M. Vignes-Adler and R. Höhler, Phys. Rev. E, 2003, 67, 021405.

17 P. Saramito, J. Non-Newtonian Fluid Mech., 2009, 158, 154161.

18 A.-L. Biance, S. Cohen-Addad and R. Höhler, Soft Matter, 2009, 5, 4672-4679.

19 M. Durand and H. A. Stone, Phys. Rev. Lett., 2006, 97, 226101.

20 M. Le Merrer, S. Cohen-Addad and R. Höhler, Phys. Rev. Lett., 2012, 108, 188301.

21 F. Heslot, T. Baumberger, B. Perrin, B. Caroli and C. Caroli, Phys. Rev. E, 1994, 49, 4973.

22 T. Baumberger and C. Caroli, Eur. Phys. J. B, 1998, 4, 13-23.

23 G. Puglisi and L. Truskinovsky, J. Mech. Phys. Solids, 2005, 53, 655-679.

24 B. Halphen and Q. NGuyen, J. Mec., 1975, 14, 3963.

25 G. Maugin, The Thermomechanics of Plasticity and Fracture, Cambridge University Press, 1992.

26 P. Coussot and G. Ovarlez, Eur. Phys. J. E, 2010, 33, 183-188.

27 L. Prandtl, Z. Angew. Math. Mech., 1928, 8, 85-106.

28 W. C. Stewart, Appl. Phys. Lett., 1968, 12, 277-280.

29 E. Bingham, Fluidity and Plasticity, McGraw-Hill, 1922.

30 G. Picard, A. Ajdari, F. Lequeux and L. Bocquet, Phys. Rev. E, 2005, 71, 010501.

31 J.-B. Salmon, A. Colin, S. Manneville and F. Molino, Phys. Rev. Lett., 2003, 90, 228303.

32 G. Katgert, M. E. Möbius and M. van Hecke, Phys. Rev. Lett., 2008, 101, 058301.

33 A. Lemaitre and C. Caroli, Phys. Rev. E, 2007, 76, 036104.

34 A. Lemaitre and C. Caroli, arXiv:0705.3122, 2007.

35 C. Gilbreth, S. Sullivan and M. Dennin, Phys. Rev. E, 2006, 74, 051406.

36 J. Lauridsen, M. Twardos and M. Dennin, Phys. Rev. Lett., 2002, 89, 098303.

37 E. Pratt and M. Dennin, Phys. Rev. E, 2003, 67, 051402.

38 R. H. Ewoldt, A. E. Hosoi and G. H. McKinley, J. Rheol., 2008, 52, 1427-1458. 